\title{
Water Quality Analysis of Village and Monitoring Well around Rembang Steam Power Plant (EIA in Central Java, Indonesia)
}

\author{
Sunik $^{1 *}$, Very Dermawan ${ }^{2}$ \\ Civil Engineering Department, Widya Karya Catholic University,East Java, Indonesia, www.widyakarya.ac.id, \\ Water Resources Engineering Department. Brawijaya University,East Java, Indonesia, http://pengairan.ub.ac.id, \\ veryderma@yahoo.com ${ }^{2}$ \\ *Corresponding author:sunik146@gmail.com
}

\begin{abstract}
Water quality sampling and monitoring of village well as a regular monitoring each quarter in a year for Rembang Steam Power Plant 2 x 315 MW was one part of EIA implementation study. This research aim to analysis the most impact occurrence that forecasted as a hypothetical important impact which is cause environmental change base on water quality sampling and monitoring from operational activity in first quarter (TW 1). Implementation method was used water sampling in 2(two) resident well and 6 (six) monitoring well in Trahan and Leran village, then the water sample was analyze in laboratory refer to the raw quality which has been set. The monitoring result on the residents wells quality was generally known that in the Trahan village residents wells (AB-1) found parameter that exceed quality standard i.e. total dissolved solids (TDS, come from river seepage and rainfall runoff), Chloride and Hardness, the high concentration some parameters, both exceed the quality standard as well as have a high critical level, generally was not caused by the operational activities of Rembang Steam Power Plant. Monitoring result from 6 (six) monitoring wells in location of Rembang Steam Power Plant was found parameters in several point that exceed the quality standard i.e. Turbidity in AB-3, organic chemical substance (KmnO4) in AB-3 and AB-7, TDS in AB-3 (monitoring wells 1), AB-4 (monitoring wells 2), AB5 (monitoring wells 3) and AB-7 (monitoring well 5), and AB-8 (monitoring wells 6). While the content of heavy metals that detected include arsenic, cadmium, lead, and selenium on AB-3.
\end{abstract}

Keywords $\longrightarrow$ water quality sampling, village well and monitoring well, impact, hypothetical important impact

\section{INTRODUCTION}

Electrical energy has a fundamental role in supporting the development of a region, in term of supporting a wide range of activities in accordance with the functions and roles of the region. Electrical energy is generally used for fulfillment needs of residental, public and social facilities (educations, healths, worships, recreations, etc), industry in a variety of scale, trade and service, and system and infrastucture areas. It can be said that the level of electrical energy sevice in a region will determine the level of development of the region. It mean that if the electrical energy requirements needs can be fulfilled, in term of the capacity and the distribution, the activities in a region which has high level of complexity can run well and fast which later affect the development of the region.

The construction purpose of Rembang Steam Power Plant $2 \times 315 \mathrm{MW}$ was to fulfill the needs of electrical energy for various interest of constructions, especially for Java, Madura, and Bali. Rembang Steam Power Plant $2 \times 315 \mathrm{MW}$ is an implementation of goverment programs in the intensification, diversication and energy conversion. In this case, the coal potential utility in Indonesia as the source of energy and reduce the dependence on petroleum. Various effects that cause by change enabling environment of Rembang Steam Power
Plant $2 \times 315$ MW activities operasional must be studied and analyzed comprehensive, accurate and precise to anticipate management and monitoring of environment impacts, so the impact which is predicted to arise can be prvented or minimalized earlier. Rembang Steam Power Plant $2 \times 315 \mathrm{MW}$ located in the Leran village and Trahan Village; subdistrict Sluke; Rembang district; Central Java Province. Power plant site was located on the North coast of Java Island, located approximately \pm $130 \mathrm{~km}$ to the East of Semarang city or more precisely at coordinates $6^{\circ} 30^{\prime}-7^{\circ} 00^{\prime}$ South latitude and $110^{\circ} 00$ '$111^{\circ} 30^{\prime}$ East longitude.

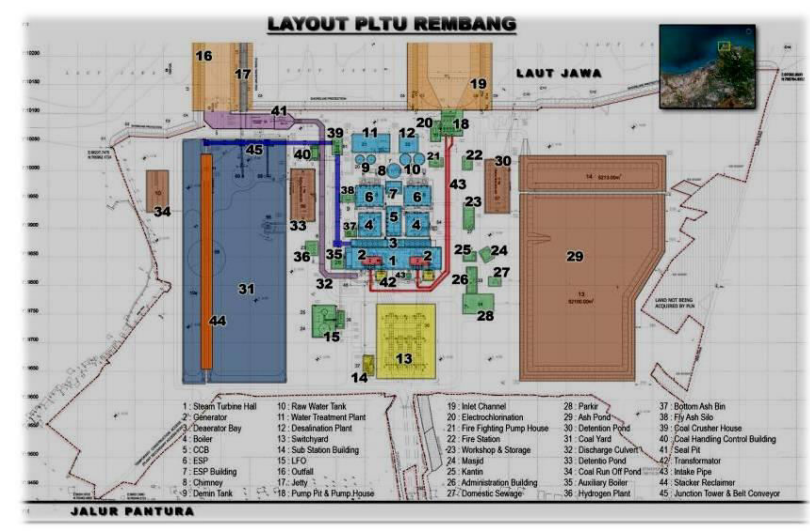

Figure 1. Layout of Rembang Steam Power Plant 2 x 315 MW

Implementation of Environmental Management and Monitoring for Rembang Steam Power Plant 2 x 315 MW First Quarter 2014 held on Month January-March 
2014 as regular monitoring agenda which is includes environmental quality sampling (water quality).

\section{METHOD}

Research was carried out in Trahan village and Leran village. Water sampling was done in 2 (two) resident well and 6 (six) monitoring well then the water sample was analyze in water quality laboratory (PJT 1 laboratory) refer to the raw quality which has been set.
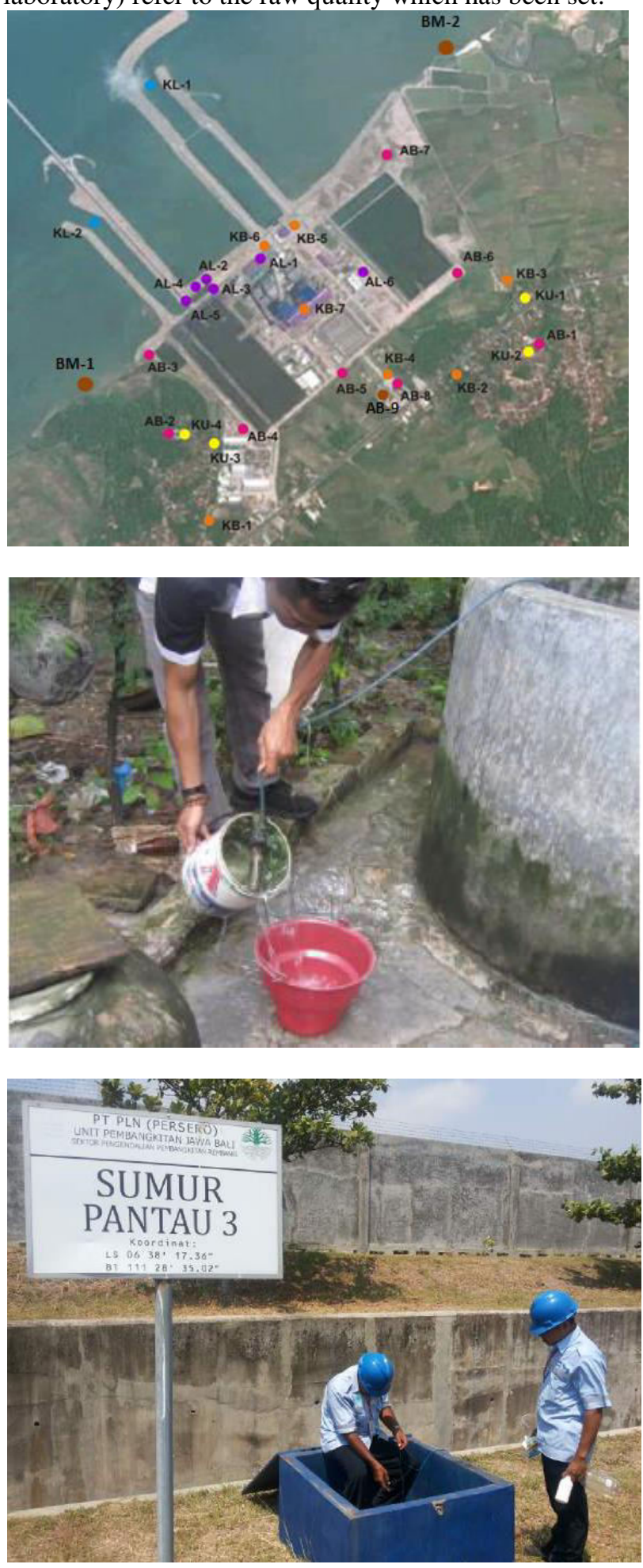

Figure 2. Water sampling for resident and monitoring well in Leran and Trahan village (AB-1 until AB-8)

The method to know water quality decrease was used Implementation (Environmental Management Implementation and Environmental Monitoring Implementation) and Evaluation as follow:

A. Implementation

1. Environmental Management Implementation :

(1) Types of managed impact

Types of managed impacts were decreased of water quality (village's wells) and groundwater quality in monitoring wells that caused by Rembang Steam Power Plant 2 x 315 MW operational caused by activities of coal and coal ash storage.

(2) Source of impact

Sources of decreased impact of groundwater quality is the overall activity or activities in operation stage. Activities operation which is give potentially impact of groundwater decreased quality was the activity of hoarding coal and coal ash. It was feared coal leachate seep into the ground so that contaminate village's wells.

(3) Management action

Management action for decrease impact of groundwater quality that has done was the stockpiling of coal at a designated place (coal stock pile and ash pond) which had been coated with geomembrane and geo-textiles and prevent spills coal off-site from coal stock pile and manufacture leachate drainage which will flow into the coal runoff pond to prevent seepage of leachate coal.

(4) Quality standard reference

Quality standard reference for decreased impact of water quality (village's wells) are standard of surface water quality that refers to the Minister of Health number 416/MEN.KES/PER/IX/1990 about conditions and water quality monitoring.

(5) Management location

Management location for decrease impact of groundwater quality was implemented in Coal Stock Pile area and the area nearby Rembang Steam Power Plant 2 x 315 MW.

(6) Management schedule

Management schedule for decreased impact of groundwater quality was implemented during the operation of generator system for Rembang Steam Power Plant 2 x $315 \mathrm{MW}$.

2. Environmental Monitoring Implementation

(1) Types of impact monitored

Types of impacts monitored was ground water quality decrease (residents wells and monitoring wells) caused by operational of Rembang Steam Power Plant 2 x 315 MW.

(2) Source of impact

Sources impact of ground water quality decrease (resident wells and monitoring wells) is the overall activity or activities in the operation phase. 
Operating activities which potentially impact the groundwater quality deterioration is hoarding of coal and of coal ash. To ascertain whether the condition of the ground water quality has decreased then the water quality monitoring is carried out at several points.

(3) Monitoring location

Monitoring location for decreased impact of water quality (wells) performed at some monitoring points as the table below.

Table 1. Monitoring locations of water quality (water in residents wells and in monitoring wells)

\begin{tabular}{|c|c|c|c|c|}
\hline No & Code & Location & Coordinate & Information \\
\hline 1 & $A B-1$ & $\begin{array}{l}\text { Bp. Kasan's well, } \\
\text { Trahan village }\end{array}$ & $\begin{array}{l}\text { BT } 111^{\circ} 28^{\prime} 53,5^{\circ} \\
\text { LS } 06^{\circ} 38^{\prime} 14,5^{\circ}\end{array}$ & $\begin{array}{l}\text { Clean Water owned by local } \\
\text { residents }\end{array}$ \\
\hline 2 & AB-2 & $\begin{array}{l}\text { Jami' Baiturahim } \\
\text { Mosque well, Leran } \\
\text { village }\end{array}$ & $\begin{array}{l}\text { BT 1110 } 28^{\prime} 19,0^{*} \\
\text { LS } 06^{\circ} 38^{\prime} 24,2^{\prime \prime}\end{array}$ & $\begin{array}{l}\text { Clean Water owned by local } \\
\text { residents }\end{array}$ \\
\hline 3 & $\mathrm{AB}-3$ & Monitoring Well 1 & $\begin{array}{l}\text { BT } 111^{\circ} 28^{\prime} 15,14^{*} \\
\text { LS } 06^{\circ} 38^{\prime} 24,2^{\prime \prime}\end{array}$ & $\begin{array}{l}\text { Clean water inside Steam Power } \\
\text { Plant }\end{array}$ \\
\hline 4 & $\mathrm{AB}-4$ & Monitoring Well2 & 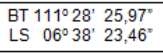 & $\begin{array}{l}\text { Clean water inside Steam Power } \\
\text { Plant }\end{array}$ \\
\hline 5 & $A B-5$ & Monitoring Well 3 & $\begin{array}{l}\text { BT } 111^{\circ} 28^{\prime} 35,02^{\circ} \\
\text { LS } 06^{\circ} 38^{\prime} 17,36^{\circ}\end{array}$ & $\begin{array}{l}\text { Clean water inside Steam Power } \\
\text { Plant }\end{array}$ \\
\hline 6 & $\mathrm{AB}-6$ & Monitoring Well 4 & $\begin{array}{l}\text { BT } 111^{\circ} 28^{\prime} 46,91^{\circ} \\
\text { LS } 06^{\circ} 38^{\prime} 07,74^{\circ}\end{array}$ & $\begin{array}{l}\text { Clean water inside Steam Power } \\
\text { Plant }\end{array}$ \\
\hline 7 & $\mathrm{AB}-7$ & Monitoring Well 5 & $\begin{array}{l}\text { BT } 111^{\circ} 28^{\prime} 40,11^{\circ} \\
\text { LS } 06^{\circ} 38^{\prime} 55,43^{\prime \prime}\end{array}$ & $\begin{array}{l}\text { Clean water inside Steam Power } \\
\text { Plant }\end{array}$ \\
\hline 8 & AB-8 & Monitoring Well 6 & $\begin{array}{l}\text { BT } 111^{1} 28^{\prime} 40,45^{\circ} \\
\text { LS } 06^{\circ} 38^{\prime} 18,23^{\circ}\end{array}$ & $\begin{array}{l}\text { Clean water inside Steam Power } \\
\text { Plant }\end{array}$ \\
\hline
\end{tabular}

(4) Environmental parameters monitored

Environmental parameters that monitored were physical parameters, chemistry, and biology that refers to the Minister of Health number 416/MEN.KES/PER/IX/1990 about conditions and water quality monitoring.

(5) Monitoring methods

Method of data collection through field observation by sampling water quality (water wells) which then analyzed in the laboratory that has been accredited nationally. Analysis of the data by using the ratio of the required quality standards.

\section{B. Evaluation}

Evaluation stage consist of trend evaluation and critical level evaluation described in chapter III (result and discussion)

\section{RESULTS AND DISCUSSION}

\section{A. Result}

Results of water quality monitoring (resident wells and monitoring wells) at each monitoring location in detail can be seen in the table below:

Table 2. Results of Water Quality Monitoring (Residents Well)

\begin{tabular}{|c|c|c|c|c|c|}
\hline \multirow{2}{*}{ No } & \multirow{2}{*}{ Parameter } & \multirow{2}{*}{ Unit } & \multirow{2}{*}{$\begin{array}{c}\text { Quality } \\
\text { Standart }\end{array}$} & \multicolumn{2}{|c|}{ Monitoring Result } \\
\hline & & & & AB-1 & AB-2 \\
\hline 1 & Temperature & ${ }^{\circ} \mathrm{C}$ & $\pm 3^{\circ} \mathrm{C}$ & 25,0 & 25,5 \\
\hline 2 & Field pH & - & $6,5-9,0$ & 7,4 & 8,0 \\
\hline 3 & Turbidity & NTU & 25 & 0,39 & 0,31 \\
\hline 4 & Odor & - & Odorless & Odorless & Odorless \\
\hline 5 & Taste & - & Tasteless & Tasteless & Tasteless \\
\hline 6 & Organic substances (KMnO4) & $\mathrm{mg} / \mathrm{l}$ & 10 & 3,1 & 2,1 \\
\hline 7 & Total Suspended Solids (TSS) & $\mathrm{mg} / \mathrm{l}$ & - & 27,9 & 13,8 \\
\hline 8 & Total Dissolved Solids (TDS) & $\mathrm{mg} / \mathrm{l}$ & 1500 & 2203 & 1333 \\
\hline 9 & Fluoride & $\mathrm{mg} / \mathrm{h}$ & 1,5 & 0,282 & 0,226 \\
\hline 10 & Chloride & $\mathrm{mg} /$ & 600 & 695 & 400 \\
\hline 11 & \begin{tabular}{|l|} 
Nitrate as $\mathrm{N}$ \\
\end{tabular} & $\mathrm{mg} / \mathrm{h}$ & 10 & 2,038 & 1,157 \\
\hline 12 & Sulfate & $\mathrm{mg} / \mathrm{h}$ & 400 & 199,6 & 0,261 \\
\hline 13 & Hardness as $\mathrm{CaCO}_{3}$ & $\mathrm{mg} / \mathrm{h}$ & 500 & 385 & 396 \\
\hline 14 & Nitrite as N & $\mathrm{mg} / \mathrm{h}$ & 1,0 & 0,030 & 0,015 \\
\hline 15 & Detergen & $\mathrm{mg} / \mathrm{h}$ & 0,5 & 0,054 & 0,039 \\
\hline 16 & Cyanide & $\mathrm{mg} / \mathrm{h}$ & 0,1 & $\mathrm{Tt}$ & Tt \\
\hline 17 & Oils andfats & $\mathrm{mg} / \mathrm{l}$ & - & $<1,9$ & $<1,9$ \\
\hline 18 & Color & $\mathrm{TCU}$ & 50 & $<0,258$ & $<0,258$ \\
\hline 19 & arsenic & $\mathrm{mg} / \mathrm{h}$ & 0,05 & $\mathrm{Tt}$ & $\mathrm{Tt}$ \\
\hline 20 & Cadmium & $\mathrm{mg} / \mathrm{h}$ & 0,005 & $\mathrm{Tt}$ & Tt \\
\hline 21 & Copper & $\mathrm{mg} / \mathrm{h}$ & - & $\mathrm{Tt}$ & $\mathrm{Tt}$ \\
\hline 22 & Total Chromium & $\mathrm{mg} / \mathrm{h}$ & - & $\mathrm{Tt}$ & $\mathrm{Tt}$ \\
\hline 23 & Iron & $\mathrm{mg} /$ & 1 & $\mathrm{Tt}$ & $\mathrm{Tt}$ \\
\hline 24 & Mercury & $\mathrm{mg} / \mathrm{h}$ & 0,001 & $\mathrm{Tt}$ & $\mathrm{Tt}$ \\
\hline 25 & Manganese & $\mathrm{mg} / \mathrm{h}$ & 0,5 & $\mathrm{Tt}$ & $\mathrm{Tt}$ \\
\hline 26 & Lead & $\mathrm{mg} / \mathrm{h}$ & 0,05 & $\mathrm{Tt}$ & $\mathrm{Tt}$ \\
\hline 27 & Selenium & $\mathrm{mg} / \mathrm{h}$ & 0,01 & $\mathrm{Tt}$ & $\mathrm{Tt}$ \\
\hline 28 & Zinc & $\mathrm{mg} / \mathrm{l}$ & 15 & Tt & $<0,0064$ \\
\hline
\end{tabular}

Information :

- The quality standard in accordance with Ministry of Health Regulation number416/MEN.KES/PER/IX/1990 about condition and water quality monitoring

- $\quad$ DL = Detection Limit

Table 3. Results of Water Quality Monitoring (Residents Well)

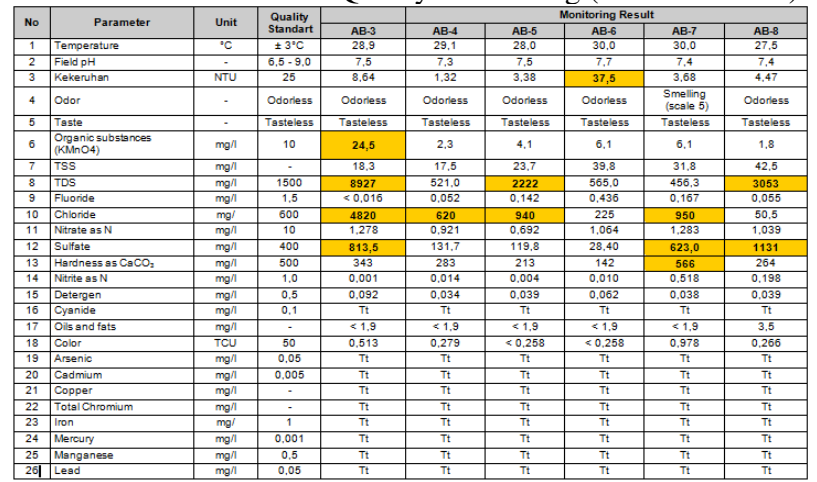

Source : Primary Data Monitoring Results Water Quality PJT I Malan3.1 g, 2014

\section{B. Trend Evaluation}

Trend evaluation was shown in Table and Figure as follow.

Table 4. Changes in Water Quality (Villager's Well) in AB-1 (Mr. Kasan's Well,Trahan Village)

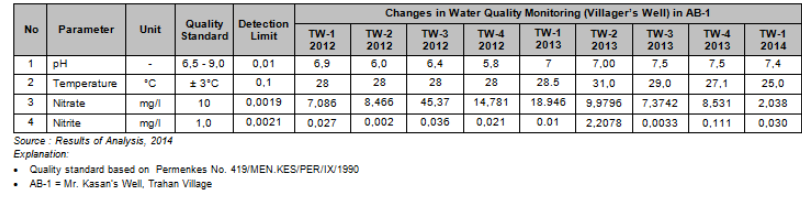

Table 5. Changes in Water Quality (Inhabitant's Well) in AB-2 (The well of Jami' Baiturahim Mosque)

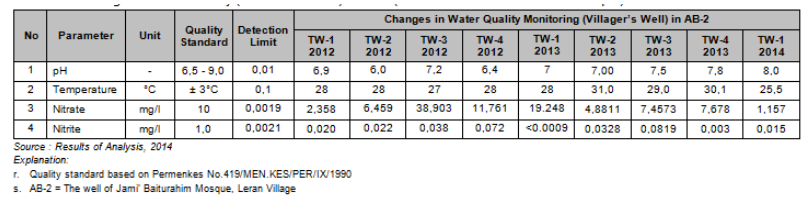




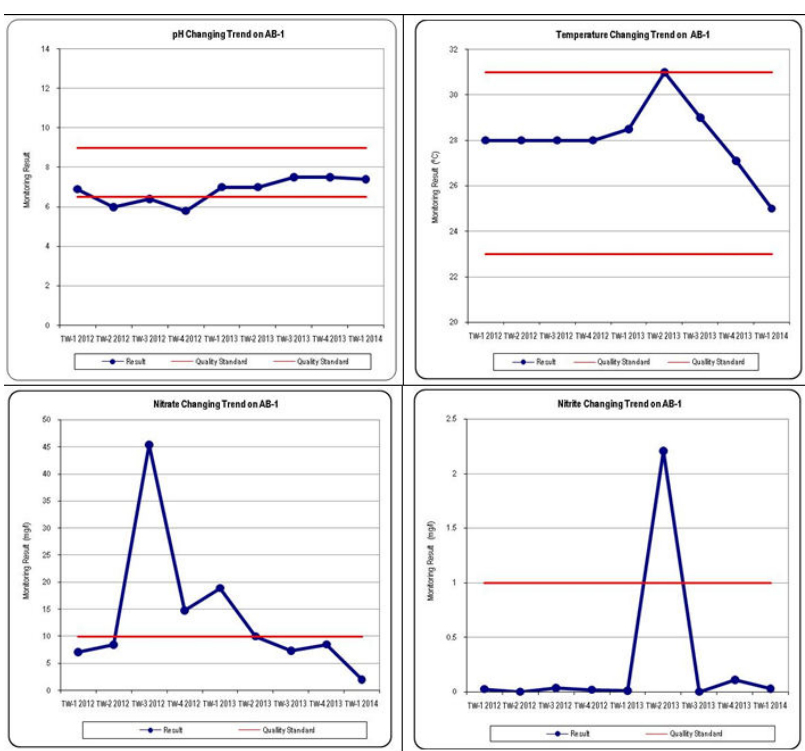

Figure 3. Diagram of Water Quality Change (resident's well) in AB-1
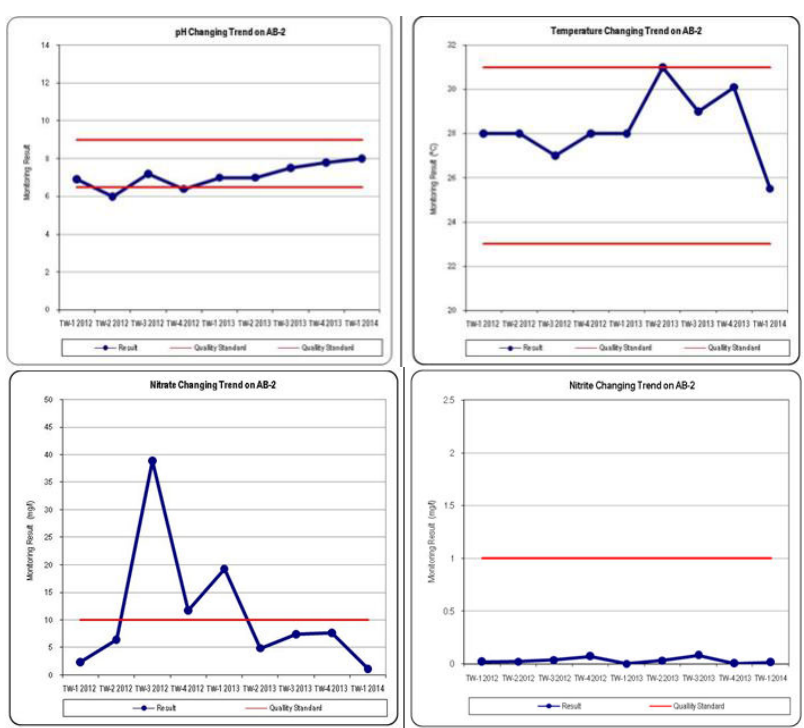

Figure 4. Diagram of Water Quality Change (resident's wells) in $\mathrm{AB}-2$

Table 6. Recapitulation on statistical result of water quality change (resident's wells)

\begin{tabular}{|c|c|c|c|}
\hline \multirow{2}{*}{ No } & \multirow{2}{*}{ Parameter } & \multicolumn{2}{|c|}{$\begin{array}{c}\text { Evaluation of Preference } \\
\text { (Linear Regression) }\end{array}$} \\
\hline & & $A B-1$ & $\mathrm{AB}-2$ \\
\hline \multirow[t]{2}{*}{1} & $\mathrm{Ph}$ & $\begin{array}{l}\text { Linear Regression Equation: } \\
y=0.17 x+6.01 \\
R^{*}=0.51\end{array}$ & $\begin{array}{l}\text { Linear Regression Equation: } \\
y=0.18 x+6.17 \\
R^{*}=0.62\end{array}$ \\
\hline & & $\begin{array}{l}\text { Which: } \\
y=p H \\
x=\text { time of messurement }\end{array}$ & $\begin{array}{l}\text { Which: } \\
y=p H \\
x=\text { time of messurement }\end{array}$ \\
\hline \multirow[t]{2}{*}{2} & Tempersture & $\begin{array}{l}\text { Linear Regression Equation: } \\
y=-0.16 x+28.88 \\
R^{*}=0.08\end{array}$ & $\begin{array}{l}\text { Linear Regression Equation: } \\
y=0.05 x+28.01 \\
R^{=}=0.01\end{array}$ \\
\hline & & $\begin{array}{l}\text { Which: } \\
y=\text { temperature } \\
x=\text { time of measurement }\end{array}$ & $\begin{array}{l}\text { Which: } \\
y=\text { tempersture } \\
x=\text { time of measurement }\end{array}$ \\
\hline \multirow[t]{2}{*}{3} & Nitrate & $\begin{array}{l}\text { Linear Regression Equation: } \\
y=-1.680 x+22.018 \\
R^{*}=0.128\end{array}$ & $\begin{array}{l}\text { Linear Regression Equation: } \\
y=-1.182 x+17.010 \\
R^{z}=0.076\end{array}$ \\
\hline & & $\begin{array}{l}\text { Which: } \\
y=\text { Nitrate } \\
x=\text { time of meassurement }\end{array}$ & $\begin{array}{l}\text { Which: } \\
y=\text { Nitrate } \\
x=\text { time of messurement }\end{array}$ \\
\hline \multirow[t]{2}{*}{4} & Nitrite & $\begin{array}{l}\text { Linear Regression Equation: } \\
y=0.041 x+0.067 \\
R^{*}=0.024\end{array}$ & $\begin{array}{l}\text { Linegr Regressions Equation: } \\
\begin{array}{l}y=-0.000 x+0.034 \\
R^{*}=0.002\end{array}\end{array}$ \\
\hline & & $\begin{array}{l}\text { Which: } \\
y=\text { Nitrite } \\
x=\text { time of measurement }\end{array}$ & $\begin{array}{l}\text { Which: } \\
y=\text { Nitrite } \\
x=\text { time of measurement }\end{array}$ \\
\hline
\end{tabular}

Source: Resulf of Data Analysis, 2014

Explanation:

- AB-1 $=$ Mr. Kasan's Well, Trahan Village

Evaluation of Preference from some water quality parameter on monitoring well located in the Steam Power Plant is shown in tables and figures below.

Table 7. Water Quality Change (Monitoring Well) in AB-

\begin{tabular}{|c|c|c|c|c|c|c|c|c|c|}
\hline \multirow[b]{3}{*}{ No } & \multirow[b]{3}{*}{ Parameter } & \multirow[b]{3}{*}{ Unit } & \multirow{3}{*}{$\begin{array}{l}\text { Quality } \\
\text { Standard }\end{array}$} & \multicolumn{6}{|l|}{ 3/SP1 } \\
\hline & & & & \multirow{2}{*}{$\begin{array}{c}\text { Detecting } \\
\text { Limit }\end{array}$} & \multicolumn{5}{|c|}{ Monitoring Result } \\
\hline & & & & & $\begin{array}{l}\text { TW-4 } \\
2012 \\
\end{array}$ & \begin{tabular}{|l|} 
TW-2 \\
2013 \\
\end{tabular} & \begin{tabular}{|l|} 
TW-3 \\
2013 \\
\end{tabular} & $\begin{array}{l}\text { TW-4 } \\
2013 \\
\end{array}$ & $\begin{array}{l}\text { TW-1 } \\
2014 \\
\end{array}$ \\
\hline 1 & \begin{tabular}{|l|} 
Dissolved Solid \\
Substance
\end{tabular} & $\mathrm{mg} / \mathrm{l}$ & 1500 & 1 & - & 5170 & 8760 & - & 8927 \\
\hline 2 & Iron & $\mathrm{mg} / \mathrm{l}$ & 1 & 0,0037 & - & $<L D$ & 0,0514 & - & Tt \\
\hline 3 & Manganese & $\mathrm{mg} / \mathrm{l}$ & 0,5 & 0,0491 & - & 2,9134 & $<L D$ & - & $\mathrm{Tt}$ \\
\hline 4 & Nitrate as N & $\mathrm{mg} / \mathrm{l}$ & 10 & 0,0019 & - & 3,6434 & 3,9862 & - & 1,278 \\
\hline 5 & \begin{tabular}{|l|l} 
Nitrite as N \\
\end{tabular} & $\mathrm{mg} / \mathrm{l}$ & 1,0 & 0,0021 & - & 0.0064 & $<L D$ & - & 0.001 \\
\hline 6 & $\mathrm{pH}$ & - & $6,5-9$ & 0,01 & - & 7,50 & 8,00 & - & 7,5 \\
\hline
\end{tabular}

- Cuality Standard based on Permenkes No.416/MEN.KES/PER/IX/1990

AB-3 = Monitoring Well 1 (Ground Water in the Steam Power Plant)

- $L D=$ Detecting Limit

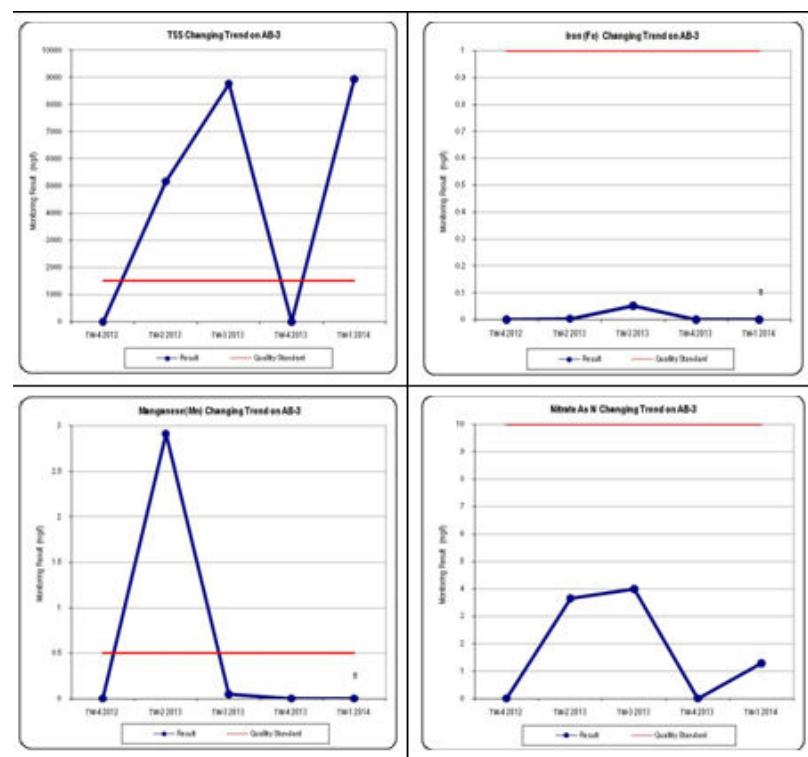

Figure 5. Diagram of water quality change (monitoring well) in $\mathrm{AB}-3$ 

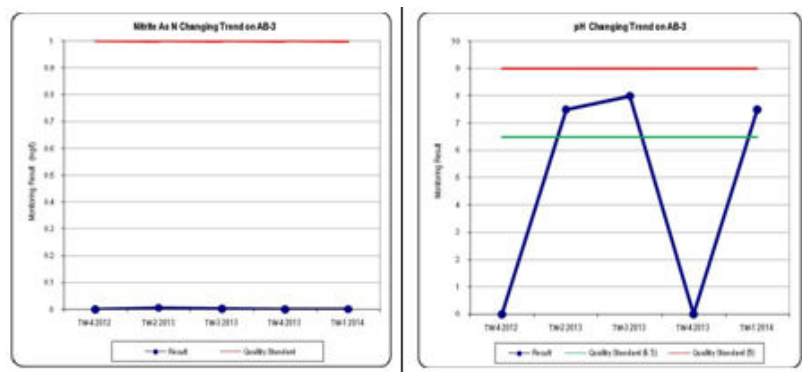

Figure 5. Diagram of water quality change (monitoring well) in AB-3 (continue)

Table 8. Water Quality Change (Monitoring well) in AB-4/SP2

\begin{tabular}{|c|c|c|c|c|c|c|c|c|c|}
\hline \multirow[b]{2}{*}{ No } & \multirow[b]{2}{*}{ Parameter } & \multirow[b]{2}{*}{ Unit } & \multirow[b]{2}{*}{$\begin{array}{l}\text { Quality } \\
\text { Standard }\end{array}$} & \multirow[b]{2}{*}{$\begin{array}{l}\text { Detecting } \\
\text { Limit }\end{array}$} & \multicolumn{5}{|c|}{ Monitoring Result } \\
\hline & & & & & \begin{tabular}{|l|} 
TW-4 \\
2012
\end{tabular} & \begin{tabular}{|l|} 
TW-2 \\
2013 \\
\end{tabular} & \begin{tabular}{|l|} 
TW-3 \\
2013 \\
\end{tabular} & $\begin{array}{l}\text { TW-4 } \\
2013\end{array}$ & $\begin{array}{l}\text { TW-1 } \\
2014\end{array}$ \\
\hline 1 & $\begin{array}{l}\text { Dissolved Solid } \\
\text { Substance }\end{array}$ & $\mathrm{mg} / \mathrm{l}$ & 1500 & 1 & 38 & 1308 & \begin{tabular}{|l|}
1082 \\
\end{tabular} & 2800 & 521 \\
\hline 2 & \begin{tabular}{|l|} 
Iron \\
\end{tabular} & $\mathrm{mg} / \mathrm{l}$ & 1 & 0,0037 & 0,028 & $\angle L D$ & 0.0179 & $<L D$ & Tt \\
\hline 3 & \begin{tabular}{|l} 
Manganese \\
\end{tabular} & $\mathrm{mg} / \mathrm{l}$ & 0.5 & 0,0491 & $<0,010$ & 0,0516 & $<L D$ & $\mathrm{Tt}$ & $\mathrm{Tt}$ \\
\hline 4 & $\begin{array}{l}\text { Nitrate } 95 \mathrm{~N} \\
\end{array}$ & $\mathrm{mg} / \mathrm{l}$ & 10 & 0.0019 & 0,207 & 0,9062 & 0.9380 & 0.718 & 0,921 \\
\hline 5 & Nitrite as N & $\mathrm{mg} / \mathrm{l}$ & 1,0 & 0,0021 & 0,421 & $<L D$ & 0,0023 & $<L D$ & 0,014 \\
\hline 6 & $\mathrm{pH}$ & - & $6,5-9$ & 0,01 & 7,9 & 7,5 & 8,00 & 8.3 & 7,3 \\
\hline
\end{tabular}

- Quality Standard is based on Permenkes No.416/MEN.KES/PER/IX/1990

- $A B-4=$ Monitoring Well 2 (Ground Water in Steam Power Plant)

- $L D=$ Detecting Limit
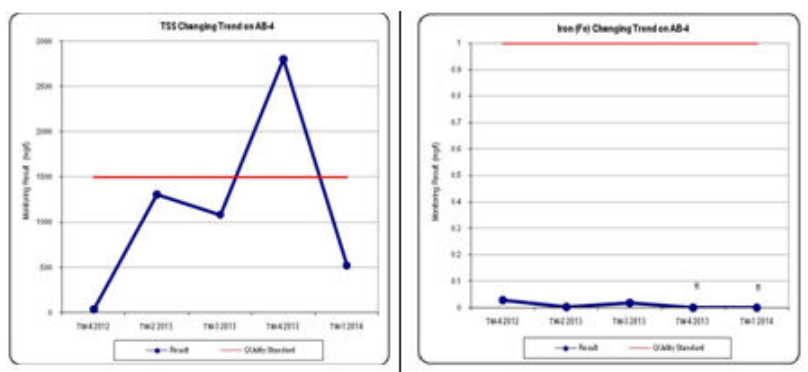

Figure 6. Diagram of water quality change (monitoring well) in $\mathrm{AB}-4$
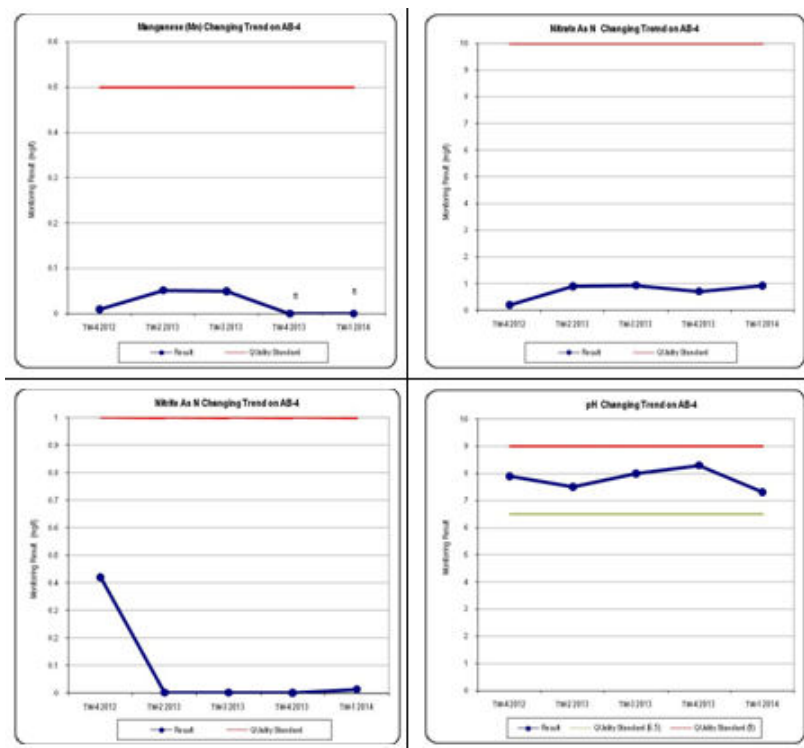

Figure 6. Diagram of water quality change (monitoring well) in $\mathrm{AB}-4$ (continue)

Tabel 9. Water quality change (monitoring well) in AB-5/SP3

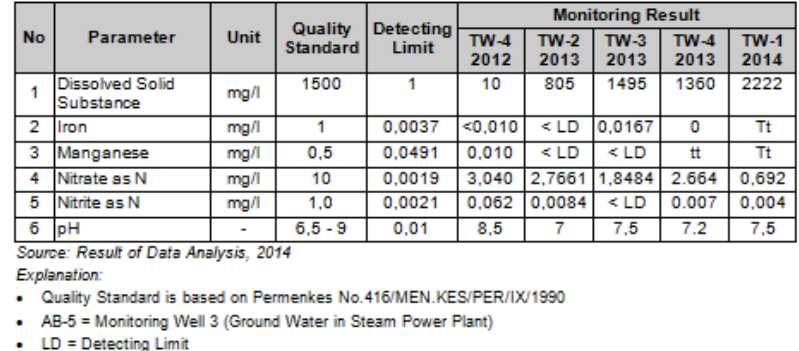

- LD = Detecting Limi

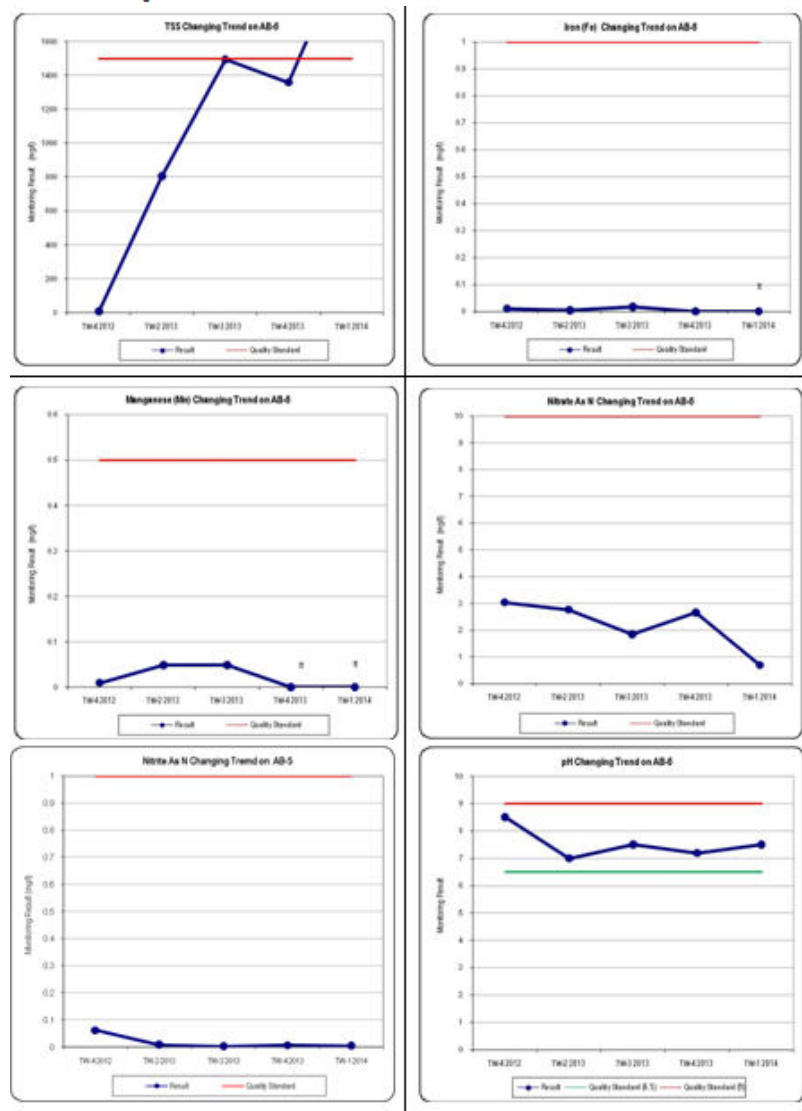

Figure 7. Diagram of water quality change (monitoring well) in $\mathrm{AB}-5$

Tabel 10. Water quality change (monitoring well) in AB-6/SP4

\begin{tabular}{|c|c|c|c|c|c|c|c|c|c|}
\hline \multirow[b]{2}{*}{ No } & \multirow[b]{2}{*}{ Parameter } & \multirow[b]{2}{*}{ Unit } & \multirow[b]{2}{*}{$\begin{array}{l}\text { Quality } \\
\text { Standard }\end{array}$} & \multirow{2}{*}{$\begin{array}{c}\begin{array}{c}\text { Detecting } \\
\text { Limit }\end{array} \\
\end{array}$} & \multicolumn{5}{|c|}{ Monitoring Result } \\
\hline & & & & & \begin{tabular}{|l|} 
TW-4 \\
2012 \\
\end{tabular} & \begin{tabular}{|l|} 
TW-2 \\
2013 \\
\end{tabular} & \begin{tabular}{|l|} 
TW-3 \\
2013 \\
\end{tabular} & \begin{tabular}{|l|} 
TW-4 \\
2013 \\
\end{tabular} & $\begin{array}{l}\text { TW-1 } \\
2014 \\
\end{array}$ \\
\hline 1 & \begin{tabular}{|l|} 
Dissolved Solid \\
Substrnoe
\end{tabular} & $\mathrm{mg} / \mathrm{l}$ & 1500 & 1 & 124 & 973 & 1199 & \begin{tabular}{|l|}
937.8 \\
\end{tabular} & 565 \\
\hline 2 & Iron & $\mathrm{mg} / \mathrm{l}$ & 1 & 0,0037 & \begin{tabular}{|l|l|}
0,044 \\
\end{tabular} & $<L D$ & 0,0171 & 0 & $\mathrm{Tt}$ \\
\hline 3 & Manganese & $\mathrm{mg} / \mathrm{l}$ & 0,5 & 0,0491 & 0,025 & $\angle L D$ & $<L D$ & 0 & $\mathrm{Tt}$ \\
\hline 4 & Nitrate as N & $\mathrm{mg} / \mathrm{l}$ & 10 & 0,0019 & 1,445 & 4,4345 & 2,8211 & 3.708 & 1,064 \\
\hline 5 & Nitrite 95 N & $\mathrm{mg} / \mathrm{l}$ & 1,0 & 0,0021 & 0,004 & 0.0118 & $<L D$ & 0 & 0,010 \\
\hline 6 & $\mathrm{pH}$ & - & $6,5-9$ & 0,01 & 7,8 & 7 & 7 & 7.8 & 7,7 \\
\hline
\end{tabular}



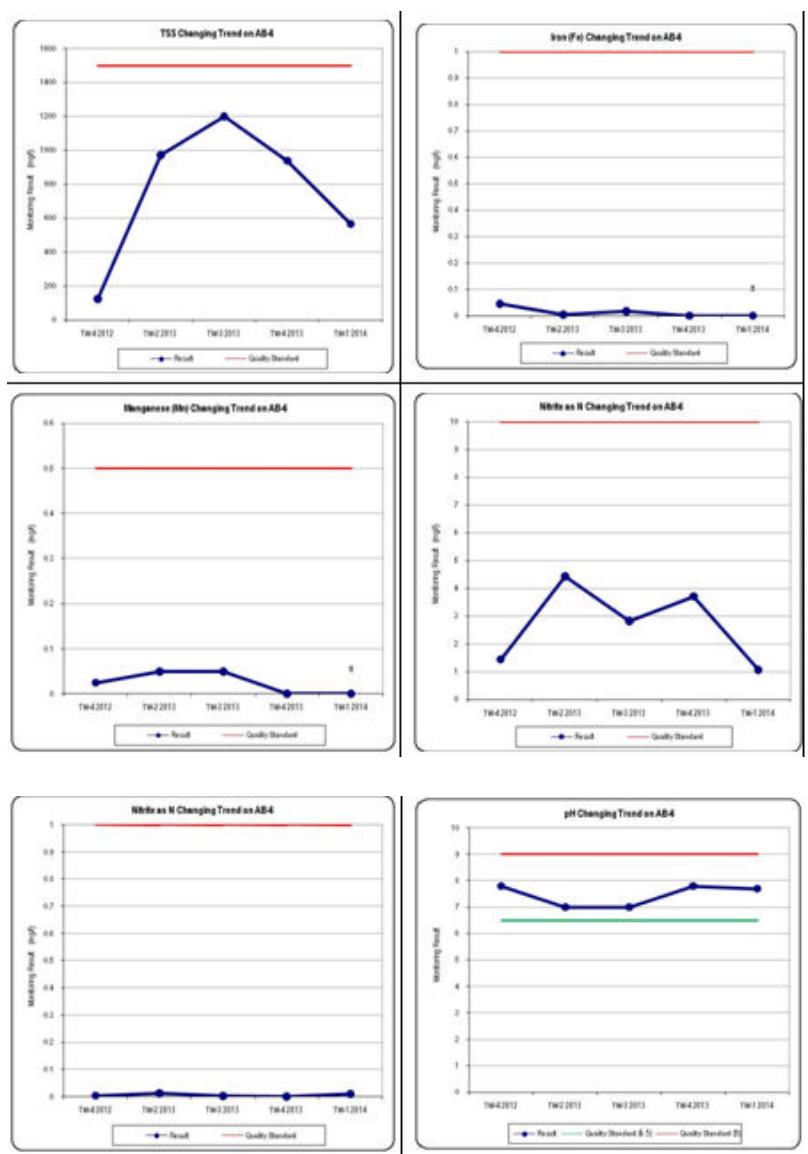

Figure 8. Diagram of Water Quality Change (Monitoring Well) in $\mathrm{AB}-6$

Tabel 11. Water Quality Change (Monitoring Well) in AB7/SP5

\begin{tabular}{|c|c|c|c|c|c|c|c|c|c|}
\hline \multirow[b]{2}{*}{ No } & \multirow[b]{2}{*}{ Parameter } & \multirow[b]{2}{*}{ Unit } & \multirow[b]{2}{*}{$\begin{array}{l}\text { Quality } \\
\text { Standard }\end{array}$} & \multirow[b]{2}{*}{$\begin{array}{c}\text { Detecting } \\
\text { Limit }\end{array}$} & \multicolumn{5}{|c|}{ Monitoring Result } \\
\hline & & & & & $\begin{array}{l}\text { TW-4 } \\
2012 \\
\end{array}$ & \begin{tabular}{|l|} 
TW-2 \\
2013 \\
\end{tabular} & \begin{tabular}{|l|} 
TW-3-3 \\
2013 \\
\end{tabular} & \begin{tabular}{|l|}
$T W-4$ \\
2013 \\
\end{tabular} & $\begin{array}{l}\text { TW-1 } \\
2014 \\
\end{array}$ \\
\hline 1 & $\begin{array}{l}\begin{array}{l}\text { Dissolved Solid } \\
\text { Substance }\end{array} \\
\end{array}$ & $\mathrm{mg} / \mathrm{l}$ & 1500 & 1 & 558 & 1523 & 2480 & 1811 & 456,3 \\
\hline 2 & Iron & $\mathrm{mg} / \mathrm{l}$ & 1 & 0,0037 & $<0,010$ & $\angle L D$ & 0.0610 & 0 & Tt \\
\hline 3 & Manganese & $\mathrm{mg} / \mathrm{l}$ & 0,5 & 0,0491 & 0,056 & $\angle L D$ & 0,1216 & $\mathrm{tt}$ & Tt \\
\hline 4 & Nitrate as N & $\mathrm{mg} / \mathrm{l}$ & 10 & 0,0019 & 0,790 & 2,9448 & 2,3808 & 2.716 & 1,283 \\
\hline 5 & Nitrite $95 \mathrm{~N}$ & $\mathrm{mg} / \mathrm{l}$ & 1,0 & 0,0021 & 0.454 & 0,0048 & 0,0094 & \begin{tabular}{|l|l|}
0.007 \\
\end{tabular} & 0,518 \\
\hline & $\mathrm{pH}$ & - & $6,5-9$ & 0,01 & 7,5 & 7 & 7,5 & 7.9 & 7,4 \\
\hline \multicolumn{10}{|c|}{$\begin{array}{l}\text { Source: Resulf of Data Analysis, } 2014 \\
\text { Explanation: }\end{array}$} \\
\hline & $\begin{array}{l}\text { Luality Standard } \\
B-7=\text { Monitoring } \\
D=\text { Detecting } L\end{array}$ & & and & . & ER & & & & \\
\hline
\end{tabular}
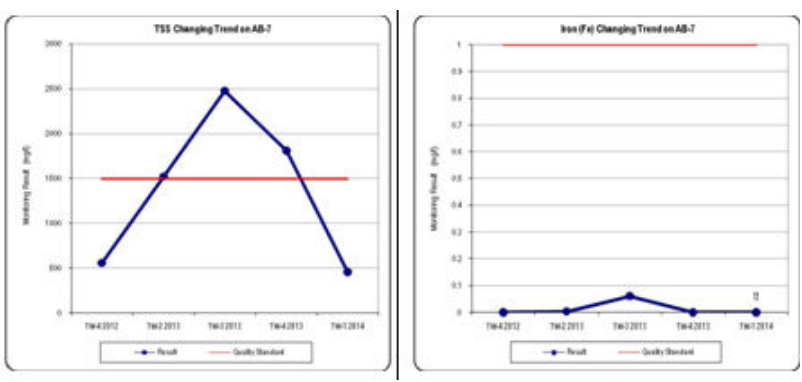

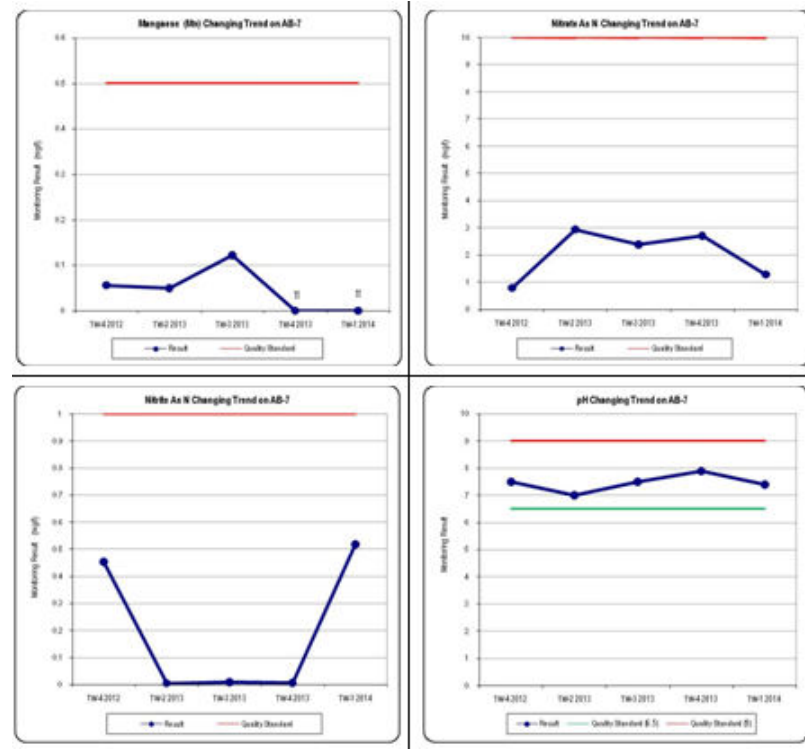

Figure 9. Diagram of water quality change (monitoring well) in $\mathrm{AB}-7$

Table 12 . Water quality changes (observation well) in AB8/SP6

\begin{tabular}{|c|c|c|c|c|c|c|c|c|c|}
\hline \multirow[b]{2}{*}{ No } & \multirow[b]{2}{*}{ Parameter } & \multirow[b]{2}{*}{ Unit } & \multirow{2}{*}{$\begin{array}{c}\text { Quality } \\
\text { Standard }\end{array}$} & \multirow{2}{*}{$\begin{array}{c}\text { Detecting } \\
\text { Limit }\end{array}$} & \multicolumn{5}{|c|}{ Monitoring Result } \\
\hline & & & & & $\begin{array}{l}\text { TW-4 } \\
2012 \\
\end{array}$ & \begin{tabular}{|l|} 
TW-2 \\
2013 \\
\end{tabular} & \begin{tabular}{|l|}
$T W-3$ \\
2013 \\
\end{tabular} & $\begin{array}{l}\text { TW-4 } \\
2013 \\
\end{array}$ & $\begin{array}{l}\text { TW-1 } \\
2014 \\
\end{array}$ \\
\hline 1 & $\begin{array}{l}\text { Dissolved Solid } \\
\text { Substance }\end{array}$ & $\mathrm{mg} / \mathrm{l}$ & 1500 & 1 & 60 & 446 & 525 & 594.5 & 3053 \\
\hline 2 & Iron & $\mathrm{mg} / \mathrm{l}$ & 1 & 0,0037 & 0,039 & \&LD & 0,0145 & 0 & $\mathrm{Tt}$ \\
\hline 3 & Manganese & $\mathrm{mg} / \mathrm{l}$ & 0,5 & 0,0491 & 0,012 & $\angle L D$ & $\angle L D$ & 0 & $\mathrm{Tt}$ \\
\hline 4 & Nitrate as N & $\mathrm{mg} / \mathrm{l}$ & 10 & 0,0019 & 0,500 & 1,2052 & \begin{tabular}{|l|l|l|l|l|l|} 
& \\
\end{tabular} & 3.73 & 1,039 \\
\hline 5 & Nitrite as N & $\mathrm{mg} / \mathrm{l}$ & 1,0 & 0,0021 & 0,003 & $\angle L D$ & $\angle L D$ & 0.013 & 0,198 \\
\hline 6 & $\mathrm{pH}$ & - & $6.5-9$ & 0.01 & 8.3 & 7 & 7,5 & 7.8 & 7.4 \\
\hline
\end{tabular}

- Quality Standard is based on Permenkes No.416/MEN.KES/PER/IX/1990 - AB-8 $=$ Monitoring Well 6 (Ground Water in Steam Power Plant) - $L D=$ Detecting Limit
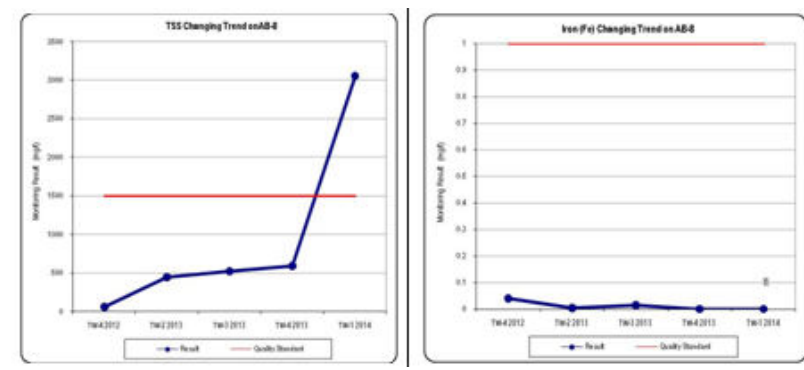

Figure 10. Diagram of water quality change (monitoring well) in $\mathrm{AB}-8$ 


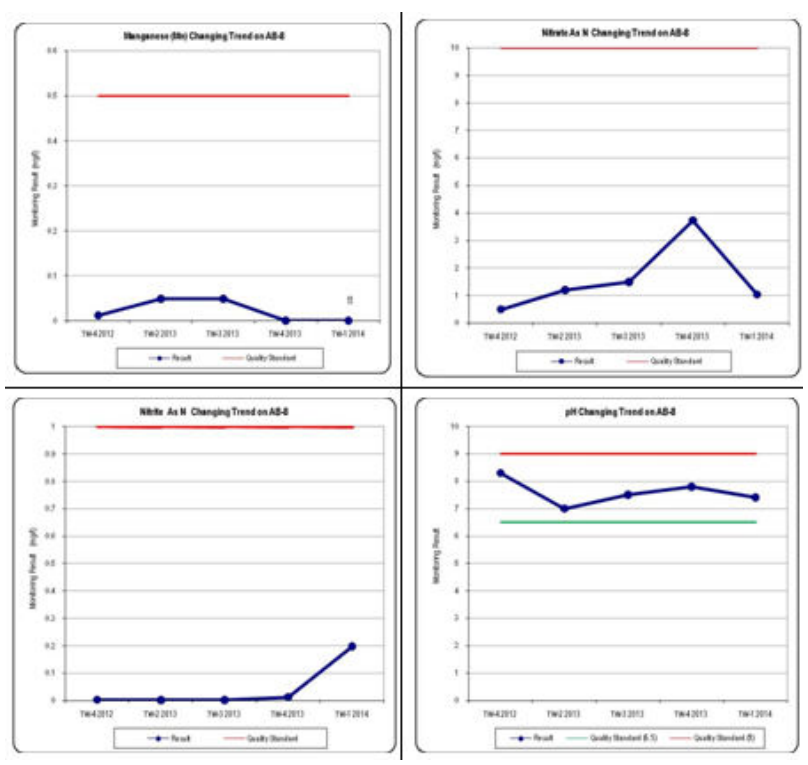

Figure 10. Diagram of water quality change (monitoring well) in $\mathrm{AB}-8$ (continue)

\section{Critical Level Evaluation}

Parameter of critical level for water quality (resident's wells) in the location AB-1 and AB-2 in some parameters have high critical level and some have exceeded the critical point (above the quality standards). Likewise, water quality (monitoring well's water) in some locations (AB-3 to AB-8) in some parameters have high critical level and some has exceeded critical level (above the quality standards). The evaluation result of the critical level of water quality (people's wells and monitoring well) for some parameters in every location is presented in the following table.

Table 13. Percentage and category of Critical Water of Water's Quality (Soil Water) at AB-1, AB-2, AB-3, and AB-4

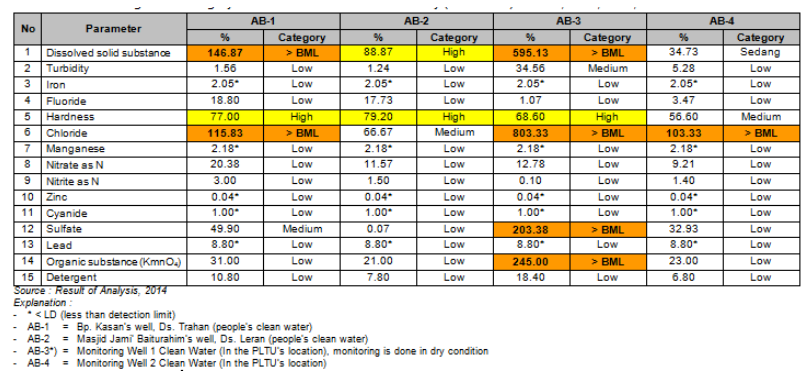

Table 14. Percentage and Category of Critical Level of Water's Quality (Soil Water) at AB-5, AB-6, AB-7, and AB-8

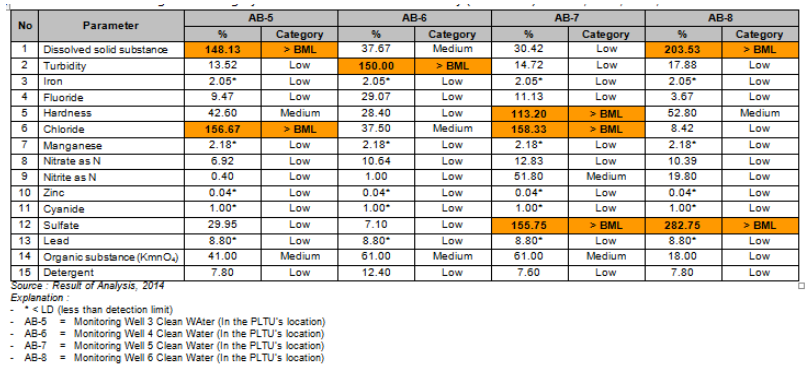

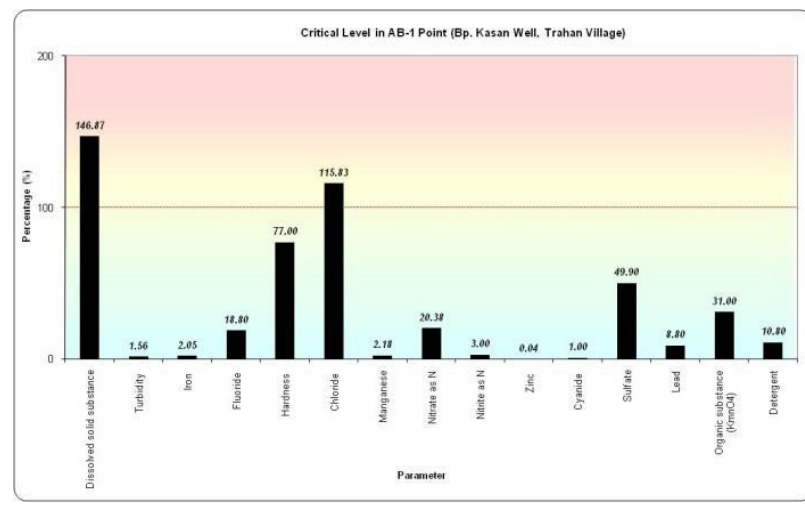

Figure 11. Graph of critical level for clean water quality at point AB-1 (Bp. Kasan's well, Trahan village for people's clean water)

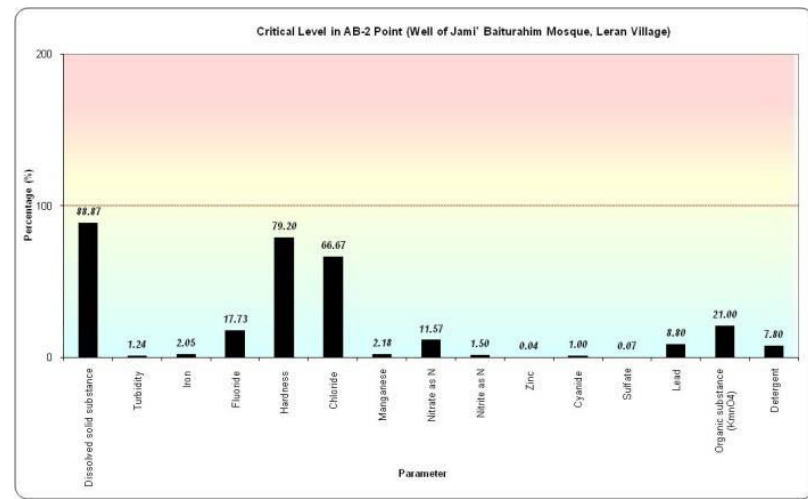

Figure 12. Graph of critical level for clean water quality at point AB-2 ( Jami' Baiturahim's Mosque well, Leran village for people's clean water)

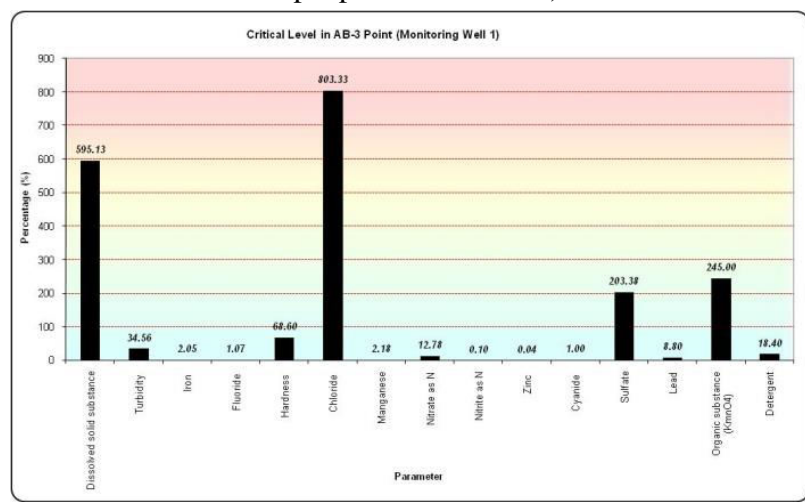

Figure 13. Graph of critical level of clean water quality at point AB-3 (monitoring well 1 of clean water in the Steam Power Plant location)

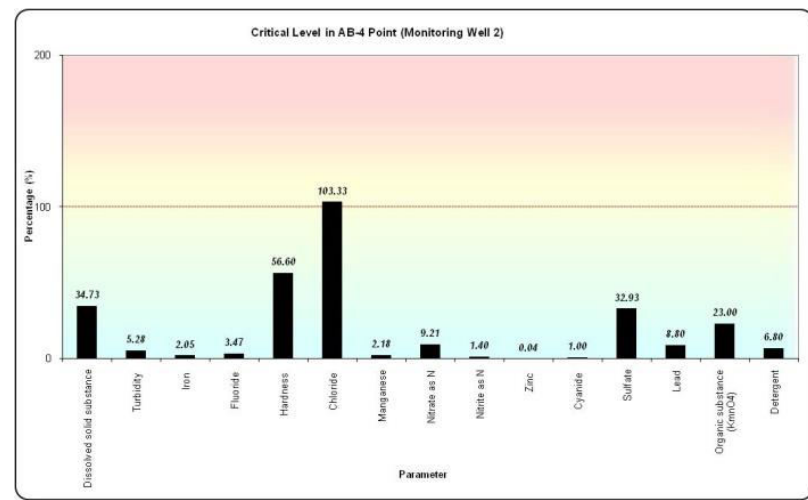


Figure 14. Graph of critical level of clean water quality at point AB-4 (monitoring well 2 of clean water in the Steam Power Plant location)

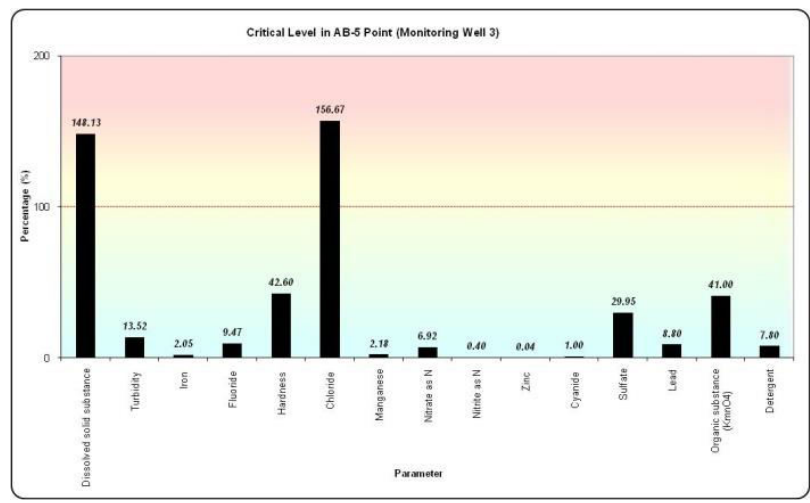

Figure 15. Graph of critical level for clean water quality at point AB5 (monitoring well 3 of clean water in the Steam Power Plant location)

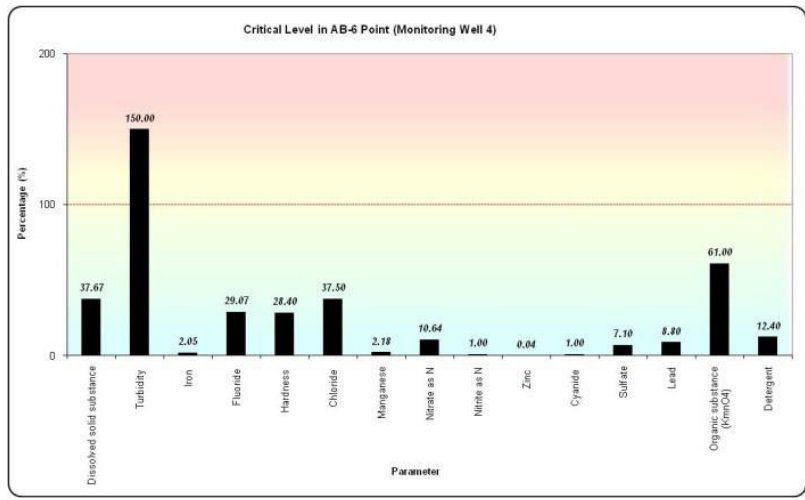

Figure 16. Graph of critical level for clean water quality at point AB-6 (monitoring well 4 of clean water in the Steam Power Plant location)

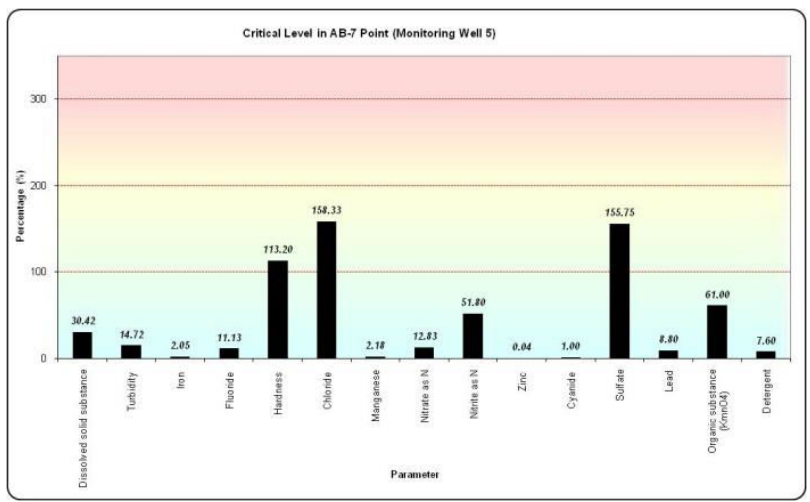

Figure 17. Graph of critical level forclean water quality at point AB-7 (monitoring well 5 of clean water in the Steam Power Plant location)

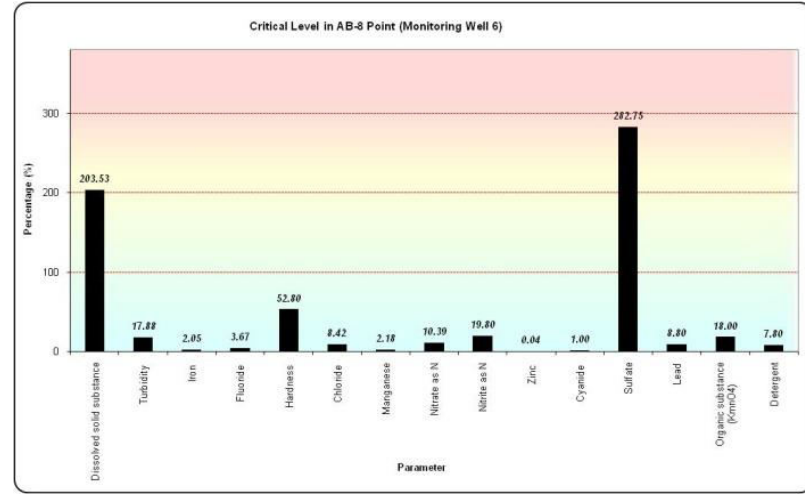

Figure 18. Graph of critical level for clean water quality at point AB-8 (monitoring well 6 of clean water in the Steam Power Plant location)

\section{Discussion}

Base on parameters of resident and monitoring well exceeding the quality standard, it can be described as follow:

1. Turbidity in AB-6

The location with turbidity level above the standard was in AB-6 (monitoring well 4). The high turbidity was related with the high Total Dissolved Solid (TDS), which affected the water clarity level. Clean water was needed to produce human consumption and industrial needs. Water turbidity was caused by suspensed substance (organic and inorganic compounds), colored organic compound, plankton and microscopic organisms. These factors were predicted cause the high turbidity in AB-6.

2. Chemical organic substance $\left(\mathrm{KmnO}_{4}\right)$ in $\mathrm{AB}-3$

Organic content shows the bacteria content. High organic substances will increase microorganism population in the water. The organic substance is easily decomposed by the bacteria using dissolved oxygen. High organic substance showed that the water has been polluted by human or animal feces, or other causes. The higher the organic substances in the water, the more obvious the water has been polluted. High organic substance in AB-3 (monitoring well 1) possibly caused by the organic material which come in the monitoring well 1.

3. Total Dissolved Solid (TDS) in AB-1, AB-3, AB-5, and $\mathrm{AB}-8$

The monitoring location with TDS which exceeding the standard were in AB-1 (Trahan village's resident well), AB-3 (monitoring well 1), AB-5 (monitoring well 3), and AB-8 (monitoring well 6). The high concentration of TDS was caused by water intrusion from environmental activities around the locations. For AB-1, high TDS mainly predicted came from the river intrusion and rainwater runoff. $\mathrm{AB}-1$ was located close to a small river, in the back of $\mathrm{Mr}$. Kasan's house. For AB-3, AB- 5, and AB-8 (monitoring wells in Steam Power Plant), high TDS can be caused by the water infiltration from the rainwater runoff. High TDS can also indicate that the water is hard. The TDS level became high value if , water hardness became high too.

4. Chloride in AB-1, AB-3, AB-4, AB-5, and AB-7 
Chlorine was often used in processing of clean water and water waste as disinfectant and oxidator. Besides, seawater also contains a lot of salt compound. The high concentration of chloride existing in AB-1 (well possessed by the society of Trahan village), $\mathrm{AB}-3$ (monitoring well 1), AB-4 (monitoring well 2), AB5 (monitoring well 3 ), and AB-7 (observation) which is located in seashore happened as there was an intrusion of seawater and the condition of land which formed from backfilled soil. It was also caused by sedimentation from seawater which made the amount of salt remaining was still high.

5. Sulphat in AB-3, AB-7, and AB-8

The sulphat concentration which exceeding the quality standard were existed in AB-3 (monitoring well 1), AB-7 (monitoring well 5), and AB-8 (monitoring well 6). The high parameter of sulphat in several monitoring wells inside the area of Steam Power Plant was thought as effect of land condition around the area which came from sedimentation process of seawater and several part of it wasformed from backfilled soil, which made salt amount of remaining was still high.

6. The Hardness of $\mathrm{CaCO}_{3}$ in AB-1 and AB-2

The high level of hardness in AB-1 (well possessed by people of Trahan village) and AB-2 (well possessed by people of Leran village) was possible due to its local geology condition. The structure of rocks in that place was limestone, which could cause the level of water hardness become relatively high. Hardness also had close correlation with TDS, which made the higher the TDS, the higher the hardness. From the result of the observation done on TDS parameter in location of $\mathrm{AB}-1$ and $\mathrm{AB}-2$, it showed that the result had been high and above the limit of standard quality, which caused the hardness on those two locations had been above the limit of standard quality of clean water recommended.

\section{Advanced Treatment}

Some advanced treatment that is advisable to do for the pioneer (or become a responsibility of the pioneer):

1. Executing maintenance on the effect that is thought will influence the condition of ground water in the area of operational activity of PLTU Rembang, according to the guide of environment's management.

Environmental treatment which are advisable to do for the society:

1. Executing process of sedimentation using filtration for solid sedimentation dissolved to the water from well which has been cooked first to fulfill the need of clean water for drinking

2. Keeping the environment's cleanliness and sanitation, especially in providing clean water for drinking through the cooperation of Local Government.

Parameter with high critical level

1. The dissolved solid (TDS) in the AB-2
The high critical level of dissolved solid for citizens' clean water in the AB-2 area (Baiturrahim mosque's well, Leran village), it is assumed because the water intrusion from the activities surrounding. The high TDS was predicted to happen because there is rain water intrusion which brings solid materials, such as soil.

2. The hardness in the AB-1, AB-2, and AB-3

The high critical level of the hardness parameter in the AB-1 (Mr. Karsan's well, Trahan village), AB-2 (well of Baiturrahim mosque, Leran village), and AB-3 (monitoring well 1) was predicted cause by intrusion from sea water and soil condition which is contain the cretaceous rocks. The water hardness content of certain minerals in the water such as ion calcium $(\mathrm{Ca})$, and magnesium $(\mathrm{Mg})$ in the form of sulphuriccarbonate. Hard water can affect the segmentation of mineral which bung up the pipeline and spigot.

\section{Prevention/Anticipation}

1. Process of segmentation should be done through filtering, in order to sediment as dissolved solid of well water and boil the water before used in order to fill the needs of clean water.

2. Implement the procedure of Steam Power Plant based on the SOP.

\section{CONCLUSION}

Base on description before, the conclusion is:

1. Water quality (residents' wells)

The observation result on the residents' wells quality was generally known that in the Trahan village residents' wells (AB-1) and AB-2 (Leran village) exceeding parameters were found, that is total dissolved solid (TDS), Chloride, and Hardness of the water. These things were predicted to be occurred because TDS was predicted to come from river water's seepage and rainwater runoff, Chloride was predicted to come from the location that is close with sea and the use of disinfectant, the water hardness was predicted to come from the geology condition of the location. The high concentration of some parameters, both exceeding the standard and having high critical level, generally was not caused by the operational activities of Rembang Steam Power Plant.

2. Water quality (monitoring well)

Result obtained from observation in six locations of monitoring wells in Rembang Steam Power Plant, some parameters which exceeding the standard were found in some spots as Turbidity in AB-6, Organic Chemical Substance (KmnO4) in AB-3, TDS in AB3 (Monitoring Well 1), AB-5 (Monitoring Well 3), and AB-8 (Monitoring Well 6), Chloride in AB-3 (Monitoring Well 1), AB-4 (Monitoring Well 2), AB5 (Monitoring Well 3), and AB-7 (Monitoring Well 
5), and also Sulfate in AB-3 (Monitoring Well 1), AB-7 (Monitoring Well 5), and AB-8 (Monitoring Well 6). The causes of the high parameter concentration are: the water turbidity was predicted to happen because of the high TDS that influence the water clarity level, Organic substances because there were some organic material which come from the intrusion to the monitoring well's water, TDS was predicted to come from rainfall run-off intrusion, Chloride was predicted to occur because of the sea water intrusion and the soil condition which once a backfill soil, and sedimentation from the sea that resulted in the high level of salt remaining, Sulfate was predicted to come from the soil condition surround the location is originally from sea sedimentation process and the Steam Power Plant location which partly from former backfill soil, thus the salt remaining is still high.

Some of the parameters which had high critical level and potential to exceed the standard are the water hardness in AB-3 (monitoring well 1) in $68.60 \%$.

Based on the observations that have been done, some advices had been made with the aim to have a better maintenance in the future and can be practically applied, particularly to reduce and prevent the environmental impacts that caused by Rembang Steam Power Plant 2 x 315 MW operational activities. The recommendation are in form of advanced treatment (which has not been summarized in Environmental Management Plan) toward the environment components which already exceeding the standard, and prevention or anticipation for the high critical level environment components as:

1. A sample test for the water quality that goes to the water area should be conducted for checking the nitrate, and to make sure that the nitrate increasing is caused from the other activities such as farming, fishery, and residents' domestic activities, not because of Power Plant activity..

2. Open the information access to the residents to accept suggestions and input related with the operational activities of Rembang Steam Power Plant which affected to the society, and then respond it quickly and accurately.

\section{Prevention/Anticipation}

Some of the parameters which have been monitored and resulted in exceeding the standard and have high critical level which happened outside Rembang Steam Power Plant area, and not because of Power Plant operational activities, some things were recommended as follows:

1. To conduct precipitation process toward filtration for precipitation of dissolved solid to the well water and the water that has to be boiled first in order to be able to use as drink water/clean water necessity.

2. To keep the environment and sanitary cleanliness, especially for the clean water supply, by doing cooperation with government district in the area. 


\section{REFERENCES}

[1] Anonym, "Implementation of Environmental Management and Monitoring Report of Rembang Steam Power Plant 2 x 315 MW", First quarter report, January March 2014, Environmental Centre Research, Brawijaya University.

[2] Environmental State Minister regulation of Indonesia Republic number 05 (2012) about type of business plan and/or activity that require Environmental Impact Assesment (EIA) analysis.

[3] Environmental State Minister regulation of Indonesia Republic number 16 (2012) about document guideline of environmental.
[4] Government regulation of Indonesia Republic number 27 (2012) about environmental permit.

[5] Health Minister regulation number 416/MEN.KES/PER/IX/1990 about conditions and water quality monitoring.

[6] Law of Indonesia Republic number 32 (2009) about protection and management of environmental.

[7] Water Quality Laboratory result number 0415-0422 S/LKA MLG/II/2014 about water quality result for resident well and monitoring well in Leran and Trahan village,2014, Jasa Tirta 1,Malang, East Java. 